\title{
The Emergence of Eating Disorders in the Context of and Comorbid with Diabetes Mellitus Type 1 and 2
}

\author{
Genevieve Dammery* and Sarah Maguire \\ Inside Out Institute, University of Sydney, Australia
}

Submission: January 06, 2020; Published: January 22, 2020

*Corresponding author: Genevieve Dammery, Inside Out Institute, Level 2 The Charles Perkins Centre, University of Sydney, Camperdown 2006, NSW, Australia

\begin{abstract}
A substantial body of literature has examined the causative factors of Diabetes Mellitus, both Type 1 and Type 2. Comorbidity between diabetes and eating disorders (EDs) have been reported in studies dating back to the 1980s. A review of the literature indicates that relatively little information is available about these comorbidities, the risk factors associated with them, the mechanisms of their development, and how to treat them appropriately. This narrative review of the literature finds that there is a concerning prevalence of EDs and disturbed eating behaviours (DEBs) in the Type 1 Diabetes Mellitus population (T1DM). Type 2 Diabetes Mellitus (T2DM) seems to be associated with some EDs and not others, and there are no studies assessing the long-term outcomes and mortality in patients with T2DM and EDs. Comorbidity of EDs and diabetes can be serious and life threatening. People with both conditions often omit insulin with the intention of inducing glycosuria, increasing the risk of physiological complications such as neuropathy, nephropathy and retinopathy and early death. A greater understanding of the correlations between comorbid diabetes and EDs should lead to better treatments. We note the potential emergence of these concurrent conditions in Australian indigenous populations. Other areas for future research are also highlighted.
\end{abstract}

Keywords: Obesity; Diabetes; Eating Disorders; Binge Eating Disorder; Anorexia Nervosa; Type 1 Diabetes; Type 2 Diabetes

Abbreviations: ED: Eating Disorder; AN: Anorexia Nervosa; ARFID: Avoidant Restrictive Food Intake Disorder; BED: Binge Eating Disorder; NES: Night Eating Syndrome; BMI: Body Mass Index; DEB: Disturbed Eating Behaviour; EDE-Q: Eating Disorder Examination Questionnaire; DSM-V: Diagnostic and Statistical Manual of Mental Disorders

\section{Introduction}

Eating disorders (EDs) are complex mental health concerns, characterised by abnormal eating behaviours and weight disturbances. Diabetes Mellitus is a metabolic disorder, which occurs in two types. Type 1 Diabetes Mellitus (T1DM) is an autoimmune disease in which the pancreas fails to produce enough insulin, and Type 2 Diabetes Mellitus (T2DM) occurs as a result of insulin resistance, often caused by lifestyle factors such as a sedentary lifestyle and obesity. Physiological consequences that may arise due to diabetes include retinopathy and neuropathy [1-3]. Importantly a diagnosis of diabetes is also associated with increased risk of developing psychological disorders such as anxiety and depression $[2,4,5]$. Comorbidity between diabetes of both types and EDs have been reported in studies dating back to the 1980s, with one study showing women with T1DM were 2.4 times more likely to develop an eating disorder than women without diabetes [6]. Evidence to date suggests that Type 1
Diabetes is more likely to precede the onset of restrictive EDs, namely Anorexia Nervosa (AN), or Avoidant Restrictive Food Intake Disorder (ARFID) [6].

Comparatively little research has been conducted into the link between EDs and T2DM. However, there is increasing evidence to suggest that Binge Eating Disorder (BED) is more prevalent in those with T2DM than those without, and that between 10 to $40 \%$ of patients with T2DM meet the diagnostic criteria for an eating disorder [7-9]. EDs that are associated with 'overeating' and subsequent weight gain - BED, Night Eating Syndrome (NES) and Bulimia Nervosa (BN) - may precede the development of T2DM although to date there are no longitudinal studies examining this directly.

The current global burden of disease associated with EDs and diabetes and is substantial. According to the Global Report on Diabetes, published by the World Health Organisation, 422 
million adults lived with diabetes in 2014, doubling the global prevalence since 1980 [10]. Globally in 2012, diabetes caused 1.5 million deaths, and high blood sugars caused an additional 2.2 million deaths [11,12]. In 2013, 1.9 million Disability Adjusted Life Years (DALYs) related to EDs [13]. With increasing instances of comorbid diabetes and EDs, the global burden of health will continue to rise unless progress is made in diagnosing, treating and reducing incidences of these conditions.

We discuss the prevalence of EDs comorbid with T1DM and T2DM, and examine risk factors for the development of, and barriers to recovery from, these comorbid diseases. We also assess current treatment options and areas for future research.

\section{T1DM and Eating Disorders}

The prevalence of EDs, and DEBs in the diabetic population is concerning. A 2015 study examining people with T1DM over 14 years indicated that the average age of onset for an eating disorder in this cohort was 18.3 years. By the age of $23,79 \%$ of the sample displayed DEBs and by 25 years old, $60 \%$ of female patients with T1DM experienced an eating disorder [14]. The average duration of the eating disorder in those with T1DM was 4.3 years, and the average time between the onset of DEBs and their remission was 6 years [14]. The incidence of DEBs and EDs continued to increase over the 14-year period of the study [14]. Two additional recent studies have reported that between $11.7 \%$ and $20 \%$ of males suffering with T1DM displayed DEBs, however, were not given a full eating disorder diagnosis. These males reported less body dissatisfaction than their female diabetic counterparts, but a higher drive for thinness when compared to their male non-diabetic peers $[15,16]$. This is an area that requires further research, as the prevalence of EDs in the male non-diabetic population continues to rise.

It has been repeatedly reported that T1DM can precede the development of an eating disorder $[3,6]$. Whilst the reason for this is likely multifactorial, a major contributor is that psychosocial factors that contribute to the development of EDs - including weight concerns, comorbid mental health conditions, and social pressures from peers - are all exacerbated by a diagnosis of T1DM in young adolescence [3]. Commonly the pathogenesis for the development of an ED in a person with T1DM involves the young person intentionally restricting their insulin for the purpose of inducing glycosuria as a means of purging calories through their urine $[1,17,18]$. This dangerous behaviour causes the body's muscle and fat stores to be broken down to produce energy, releasing ketone bodies and resulting in a shift in $\mathrm{pH}$ balance in the body, known as diabetic ketoacidosis [15]. This set of behaviours is often preceded by body dissatisfaction caused by weight gain that frequently results from insulin administration. Dietary restriction, a normalised approach to treating T1DM is theorised to also contribute to the development of Disturbed Eating Behaviours (DEBs).
Although DEBs may appear less severe than a diagnosable eating disorder, it has been noted that their duration is longer [17], and this is possibly due to the fact that they are more likely to be unidentified in a clinical setting, and therefore be left untreated. If clinicians are not aware of and do not address DEBs and insulin misuse, numerous unfavourable hyperglycaemia induced consequences may arise including microvascular, neuropathic and retinopathic conditions [17]. Prolonged insulin restriction and subsequent malnutrition also leads to physiological complications commonly seen in patients with EDs- osteoporosis, heart conditions, fertility problems, renal complications, electrolyte abnormalities and gastrointestinal disturbances $[19,20]$.

\section{T2DM and Eating Disorders}

T2DM appears to be associated with some eating disorder diagnoses and not others. A number of studies have shown that that people with a diagnosis of BN and BED are at increased risk of developing T2DM. One study conducted over 16 years showed an increased risk of T2DM in patients suffering from BED and BN. It was found that by the end of the 16-year period, onethird of patients who began the study with BED had developed T2DM. Another study indicated that nearly $90 \%$ of patients suffering with both T2DM and EDs develop the eating disorder prior to their diabetes diagnosis [21]. Further, that those with T2DM would restrict oral medications intentionally for weight control purposes, increasing their risk of long-term physiological complications [9]. Importantly, the risk of developing T2DM in patients suffering with BN decreased as they received treatment for their eating disorder [9]. There is no research to date to suggest a correlation between restrictive type EDs, such as AN and ARFID, and T2DM. Also, of concern is the higher incidence of subclinical binge eating in T2DM, which studies suggest affects between $7-20 \%$ of adults [7,22]. As DEBs, such as subclinical binge eating, are associated with poor metabolic control, early interventions for individuals presenting with DEBs are necessary to slow or prevent the development of diabetes related physical complications.

\section{Mortality in Patients with T1DM and Eating Disorder}

\section{Behaviours}

It has been repeatedly demonstrated that insulin restriction worsens diabetes related complications and increases the chances of early death [2]. Over an 11-year study period, it was found that prolonged insulin restriction tripled the risk of patient death and lowered the age of death [2]. A study examining concurrent anorexia nervosa (AN) and T1DM found an alarming increase in the mortality rate in this group; those who suffered with comorbid T1DM and AN was 34.8\%, compared to 2.5\% in those who suffered with only T1DM and $6.5 \%$ in AN patients [23]. This study did not provide insight into the full extent of mortality for comorbid EDs and T1DM as it was limited to an eating disorder diagnosis of AN 


\section{Current Research in Diabetes \& Obesity Journal}

with T1DM, and AN only represents a small proportion of eating disorder cases.

\section{Mortality in patients with T2DM and eating disorder behaviours}

No research was found that assessed mortality in patients with T2DM and EDs.

\section{Risk factors for the development of eating disorders in individuals with T1DM}

There are several identified risk factors for the development of EDs in patients with T1DM.

Rapid weight gain in patients following the commencement of insulin therapies may cause psychological distress in patients, reducing self-esteem and contributing to poor body image [24] By inference, the current treatments for T1DM may inadvertently be contributing to an increased incidence of concurrent EDs since insulin administration often results in weight gain in those with T1DM and T2DM. Suggested means of reducing insulin-related weight gain, such as increasing exercise and restricting particular foods from the diet, are impractical and counter-productive in treating eating disorder patients as these often form part of the core symptoms of the eating disorder and encouraging an increase in these could increase the intensity of the illness, therefore other treatments for reducing insulin-related weight gain are needed. Heightened focus on meal plans, and dietary restrictions associated with treating T1DM, may also be contributing to an increased incidence of concurrent EDs and T1DM, since these treatment methods can produce an unhealthy focus and obsession with food, diet and weight in patients with diabetes. Whether this could trigger EDs deserves investigation; we could find no studies directly examining the contribution of treatments for T1DM on onset for an ED. It certainly makes the detection of EDs more difficult since rigidity and preoccupation with food in individuals with diabetes is common and even encouraged [25].

Concurrent mental health conditions such as depression and anxiety are also common in young patients with diabetes [2628] - as they are in patients with EDs [29-31] - and can have a considerable impact on eating patterns and patient appetite. It has been consistently reported that there are higher rates of depression and anxiety in those with T1DM, and EDs are associated with high rates of psychiatric comorbidities [4]. Anxiety is commonly experienced in those who are newly diagnosed with T1DM as adults and may precede the development of an eating disorder [32]. Furthermore, insulin restriction in patients with T1DM has been reported to be used by some as a self-harming behaviour [33], highlighting the fact that patients are aware of the physical consequences of insulin omission and restriction. Selfmanagement of T1DM may be compromised in patients who are suffering from depression, anxiety and EDs, and therefore may require intense treatment [32]. If other mental health issues, such as depression and anxiety precede eating disorder development, treating these other mental health conditions may reduce onset of DEBs, and subsequent eating disorder development.

The age at which a patient is diagnosed with T1DM may also play a role in the development and severity of EDs. Takii and colleagues found that female patients who were diagnosed with T1DM between the ages of 7 and 18 were at significantly higher risk of developing an eating disorder [3]. Another study conducted in 2015 found that the average age that women were diagnosed with diabetes was 11.9 years old, and the average time between diabetes diagnosis and eating disorder development was 4.76 years [4]. Patients who are diagnosed with T1DM prior to the onset of puberty may be less likely to develop an eating disorder, however the reasoning for this is currently unknown. It has been hypothesised that a diagnosis of diabetes during puberty increases the risk of eating disorder development, as these young adolescents are already facing substantial hormonal, physical and psychosocial changes [3].

\section{Risk Factors for the Development of T2DM in Individuals with Eating Disorders}

It is currently understood that BED and BN may precede development of T2DM [9], at least in certain age groups, and consequently it is important to understand the psychological risk and maintenance factors involved in BED and BN, so that these illnesses can be treated before T2DM develops. Risk factors pertaining to $\mathrm{BN}$ and BED development include depressed mood and generalised anxiety [34], rigid and obsessive thinking patterns, family dysfunction, and body dissatisfaction [35]. Dissociative behaviours during a period of binge eating in patients with $\mathrm{BN}$ have been described as protective coping mechanism from emotional distress [36]. This dissociative "immersion" needs to be assessed in patients with BED during a bingeing period [36]. Both BED and BN are characterised by the consumption of large amounts of food in a short period of time, resulting over time in weight gain. In BN the excess caloric intake consumed during a binge eating episode is offset, at least in part, by compensatory behaviours that mark the illness: purging, excessive exercise, restrictive caloric periods and laxative abuse [37]. In BED there is little or no compensation following a period of binge eating, and as a result weight gain is more likely. As such, it has been reported that patients with BED have significantly higher BMIs than patients with BN [37].

Although it is understood that binge eating and obesity are associated with developing T2DM, the potential impact of other eating disorder behaviours such as restrictive eating and disturbed eating patterns on increased risk of developing T2DM have had almost no investigation. One study assessing the association between eating patterns and T2DM development, 
found that frequently skipping breakfast was associated with a $50 \%$ higher risk of developing T2DM [38]. This study included only men aged 40-75 years old. An opportunity exists to replicate this study in different age and gender populations, and particularly in populations more susceptible to EDs.

It has been reported that the average age of women and men with BN and T2DM is 38 and 44.7 years respectively, and the average age for women and men with BED is approximately 41.2 and 46.2 years respectively. One study examining binge eating and weight loss in overweight patients with T2DM showed that older patients aged between 45 and 76 years old reported low incidences of binge eating behaviours [39]. There is a high association with anxiety and depression in individuals with T2DM, with the prevalence of depression in those with diabetes being higher than in the general population [40]. The cause for the correlation between depression and diabetes is unknown, however it has been hypothesised that mental distress, depression and anxiety are a result of the strain of living with a chronic condition [25], and is a response to the physiological effects of living with T2DM [26]. Several studies have also indicated that depression may precede the development of T2DM [24], as many common symptoms of depression - sedentary lifestyle, smoking, and metabolic disturbances brought about by depression are also risk factors in the development of T2DM [26]. As both depression and EDs can precede diabetes development, it is important that patients who are suffering with these comorbid psychiatric conditions are screened for poor metabolic control. The clinical implication of concurrent depression, EDs and T2DM may be poor adherence to recommended treatments [25].

\section{Mechanisms for the Development of Eating Disorders in Patients with T1DM}

Undiagnosed T1DM can result in rapid, uncontrolled weight loss in patients, often met with praise and encouragement from family and friends of patients [41]. Once diagnosed with T1DM and treated with insulin, patients often gain weight, resulting in emotional distress in the individual, and attracting a negative response from the individual's peers, who had previously praised their weight loss [41]. Individuals who have been recently diagnosed with T1DM report feelings of not belonging, loss of control [41], and shame [24]. This emotional distress, coupled with body dissatisfaction, perfectionistic traits and over- evaluation of eating and food [42] can lead to individuals restricting insulin, and engaging in other maladaptive behaviours, such as restrictive eating, binging and purging $[34,35]$.

\section{Possible Mechanisms for Concurrent T2DM and Eating Disorders}

Currently there is no certainty around the mechanisms for the development of T2DM in patients suffering from EDs. What we do know is that previously discussed risk factors such as dysregulated mood, rigid and obsessive thinking patterns, family dysfunction and body dissatisfaction may contribute to BED and BN development [34,35]. When binge eating is used as a coping mechanism, for many people [37] substantial weight gain may follow, resulting in obesity and eventually T2DM development. If treated with insulin, a patient with T2DM may gain additional weight [15]. This unwanted and uncontrolled weight gain can cause higher levels of distress in individuals, leading them to omit or restrict their insulin intake [43]. Individuals may also engage in periods of food and insulin restriction followed by binge eating and insulin overdosing. Consequences of these actions include higher mortality rates, and retinopathy and neurological, kidney and microvascular complications. These aspects of concurrent T2DM and EDs require further research.

\section{Identified Barriers to Recovery in Patients with T1DM}

Patients suffering from comorbid EDs and diabetes do not respond positively to standard eating disorder treatments, showing lower rates of limited and complete recovery than eating disorder patients without Diabetes Mellitus [44]. One study showed that, in women with T1DM, 71\% of women with DEBs experienced recurrence of behaviours after remission. Amongst those with diagnosed EDs, $60 \%$ experienced a recurrence of their eating disorder after remission [19]. Whilst there remains no definitive explanation for the poor outcomes and recurrence of EDs and DEBs, the role of social identity in recovery has been considered. Currently, patients with diabetes who restrict insulin for weight loss use the term 'Diabulimia' to describe their condition, however it is not a clinically recognised diagnosis. The term 'Diabulimia' is unfamiliar to many clinicians and medical professionals, and patients report that because of this, clinicians lacked empathy when treating them [44] and perhaps the specific skillset to target the two illness groups together. Consistently, clinicians have reported difficulties in treating the comorbid conditions, citing a lack of education and training on treating the dual diagnosis, patient resistance to treatment, and uncertainty regarding when insulin misuse becomes a disordered behaviour as major barriers to their ability to appropriately treat patients $[45,46]$. Without a formal diagnosis and sense of social identity, individuals with diabetes and concurrent EDs report not feeling supported by clinicians, and lack a sense of acceptance and validation from their non-diabetic peers [44], as well as a lack of formal treatment guidelines for clinicians to follow [46]. One possible inference may be that, without a formal diagnosis specific to comorbid EDs and diabetes, sustained recovery is lower and clinical outcomes will continue to be poor.

\section{Identified Barriers to Recovery in Patients with T2DM}

Treating comorbid T2DM and EDs is difficult. Often eating disorder treatments involve weight restoration and nutritional rehabilitation as the first line of treatment; however, patients with 
BED and T2DM may be clinically obese, with weight reduction a clinical objective. T2DM symptoms are often better managed once the patient reduces their weight [47], however encouraging weight reduction may hinder eating disorder recovery. BED is a relatively newly recognised illness, only being acknowledged by the Diagnostic and Statistical Manual of Mental Disorders, 5th edition (DSM-V), and therefore may be unfamiliar to many clinicians who have experience treating AN and BN. BED is associated with other concurrent psychiatric disorders such as personality and affective disorders [48], which may complicate treatment of BED and T2DM.

\section{An Additional Australian Problem: Comorbid Eating Disorders and Diabetes in Indigenous Communities}

The rates of diabetes in indigenous Australian populations are 6 times higher than those of other Australians [49], and one study has demonstrated that $46 \%$ of indigenous youths in Northern Australia were overweight or obese [50]. Only a handful of studies have been conducted into eating disorder patterns and body image disturbances in indigenous populations. In 2005 it was found that indigenous Australian adolescents engaged in more strategies to lose weight when exposed to media images [51]. In indigenous communities in Australia, suicide and self-harm are a substantial issue, with suicide rates among indigenous people in the Kimberly region being some of the highest globally [52]. Of concern is that increasing Internet and media exposure will expose indigenous youth with diabetes to insulin restriction as a means of self-harm and as a strategy for altering their bodies. It has been suggested by McCabe and colleagues that media exposure of indigenous youth increased body dissatisfaction, prompting participation in strategies to alter their bodies [51]. It has also been suggested that urbanisation of rural areas has increased the risk of eating disorder development in young women [53]. Although previously indigenous youth were exposed to comparatively fewer media influences than their non-indigenous counterparts, when they were exposed, they responded more negatively. Due to the fact that body image concerns are present in indigenous communities, and disturbed eating patterns are equal to, if not more prevalent in indigenous populations when compared to non-indigenous populations [54], there is potential for a rise in EDs, especially comorbid with T2DM.

\section{Approaching Treatment of Comorbid Eating Disorders and Diabetes}

Recommended treatment for a comorbid eating disorder and diabetes is multidisciplinary including a psychologist, potentially psychiatrist, a dietician and an endocrinologist. Challenges arise when treating these comorbid conditions, as both T1DM and T2DM require different interventions, as do different EDs. Standard methods used for assessing and diagnosing EDs, such as The Eating Disorder Examination Questionnaire (EDE-Q), may not be sufficient for assessing an eating disorder in the context of diabetes. The EDE-Q and other validated instruments make no mention of behaviours such as insulin restriction, a characteristic feature of individuals with T1DM and T2DM, in particular in the presence of an eating disorder. As a result, this dangerous behaviour may remain undetected in individuals with diabetes who also suffer from EDs. The introduction of several screening tools - the Eating Problems Survey - Revised (DEPS-R), Screen for Early Eating Disorder Signs (SEEDS), and mSCOFF have created means for clinicians to enquire about disordered behaviours in diabetic individuals. These questionnaires, whilst useful in identifying behavioural risk factors, make direct mention of DEBs such as insulin restriction, and as such may actually introduce the young person being screened to DEBs that they had not previously considered, having a counterintuitive effect.

Nutritional rehabilitation is often the first, and most critical, step in eating disorder treatment. Together with nutritional rehabilitation, in patients with diabetes it is important to restore metabolic control to reduce the risk of diabetes related physical complications. There are few clinicians with expertise in treating both diabetes and EDs. A DSM recognised diagnosis of EDs comorbid with Diabetes Mellitus ('Diabulimia') may help sufferers to feel a sense of social identity and will expose and familiarise clinicians with the condition. The requirement for clinicians working either with patients with either EDs or diabetes to obtain training to aid in the screening and recognition of both illnesses will help in early detection and intervention.

\section{Directions for Future Research}

A number of opportunities for future research concerning comorbid EDs and Diabetes Mellitus have been identified in this paper. These include:

i. Developing a better understanding of the links between EDs and T2DM and establishing the mechanisms that underpin the development of T2DM in patients with BED, BN, and other EDs. A testable model for the development of T2DM in patients with EDs has not yet been established and would assist both clinicians and patients to understand the underlying mechanisms of these concurrent disorders. Given the global growth in Diabetes Mellitus this should be a high priority.

ii. Currently there is no research that assesses mortality in patients with T2DM and EDs. More quantitative data relating to insulin restriction or complete omission is required to assess mortality and morbidity in patients with Diabetes Mellitus and EDs, especially in those suffering with T2DM.

iii. It has been implied that the current treatments for T1DM and T2DM, including the strict focus on food and meal plans, and strict dietary restrictions may be leading to an unhealthy focus on food in patients, and therefore may be contributing to incidences 
of EDs in those with T1DM and T2DM. In addition, weight gain caused by insulin is a frequent cause of distress in patients with Diabetes Mellitus. Introducing insulin analogues as a first line of treatment for Diabetes Mellitus in patients with EDs, in place of Neutral Protamine Hagedorn (NPH) insulin, may be considered as a treatment option that removes this cause of distress.

iv. Only one study was found that assessed the impact of the age on T1DM diagnosis and the development and severity of EDs. Further research is required to establish whether age is a risk factor in those with T1DM, and whether a T1DM diagnosis in early childhood reduces the chances of eating disorder development. As BED is more common in older populations, an understanding of whether BED, BN and comorbid T2DM is also needed.

v. Understanding poor outcomes in patients with EDs and diabetes is necessary, as currently there are high rates of recurrence in these patients. Further research into the correlations between social identity and poor adherence to treatment are necessary to improve patient outcomes.

vi. Australia rural and indigenous populations show higher levels of obesity and diabetes, and therefore may be at higher risk for developing EDs. An enhanced understanding of the concurrence of EDs and Diabetes Mellitus in Australian indigenous communities is needed, as well as understanding the links between body image, self-harm and these conditions, as well as whether diabetes-related distress is a common trigger for insulin restriction in these communities.

\section{Conclusions}

Individuals with Type 1 diabetes are highly susceptible to developing EDs, and those with BED and BN are at risk of developing Type 2 Diabetes. Insulin omission is a common, dangerous behaviour, which increases the risk of diabetes related complications. The strategies currently used to manage T1DM may be inadvertently contributing to DEBs and EDs, as well as making detection of EDs difficult. Measures need to be taken to ensure that patients with diabetes are screened for disturbed eating behaviours, and clinicians need to be trained to treat comorbid EDs and diabetes. Further research is required to establish prevalence of EDs in indigenous communities, and to establish better treatments in order to improve patient outcomes.

\section{Acknowledgements}

Genevieve Dammery wishes to acknowledge the support of the University of Sydney, Charles Perkins Centre Summer Research Scholarship.

\section{References}

1. Colton P, Olmsted M, Daneman D, Rydall A, Rodin G (2004) Disturbed eating behavior and eating disorders in preteen and early teenage girls with type 1 diabetes: a case-controlled study. Diabetes care 27(7): 1654-1659.
2. Goebel-Fabbri AE, Fikkan J, Franko DL, Pearson K, Anderson BJ, et al. (2008) Insulin restriction and associated morbidity and mortality in women with type 1 diabetes. Diabetes care 31(3): 415-419.

3. Takii M, Uchigata Y, Kishimoto J, Morita C, Hata T, et al. (2011) The relationship between the age of onset of type 1 diabetes and the subsequent development of a severe eating disorder by female patients. Pediatr Diabetes 12(4 Pt 2): 396-401.

4. Allan J (2015) Understanding poor outcomes in women with type 1 diabetes and eating disorders. Journal of Diabetes Nursing 19: 99-103.

5. Kenardy J, Mensch M, Bowen K, Green B, Walton J, et al. (2001) Disordered eating behaviours in women with Type 2 diabetes mellitus. Eating behaviors 2(2): 183-192.

6. Jones J, Lawson ML, Daneman D, Olmsted M, Rodin G (2000) Eating disorders in adolescent females with and without type 1 diabetes: cross sectional study. BMJ 320(7249): 1563-1566.

7. Allison KC, Crow SJ, Reeves RR, West DS, Foreyt JP, et al. (2007) Binge eating disorder and night eating syndrome in adults with type 2 diabetes. Obesity (Silver Spring) 15(5): 1287-1293.

8. Herpertz S, Albus C, Lichtblau K, Kohle K, Mann K, et al. (2000) Relationship of weight and eating disorders in type 2 diabetic patients: a multicenter study. Int J Eat Disord 28(1): 68-77.

9. Raevuori A, Suokas J, Haukka J, Gissler M, Linna M, et al. (2015) Highly increased risk of type 2 diabetes in patients with binge eating disorder and bulimia nervosa. Int J Eat Disord 48(6): 555-562.

10. (2016) Organization WH. Global Report on Diabetes, p. 8.

11. Thorburn AW (2005) Prevalence of obesity in Australia. Obesity reviews: an official journal of the International Association for the Study of Obesity 6(3): 187-189.

12. Whiteford HA, Degenhardt L, Rehm J, Baxter AJ, Ferrari AJ, et al. (2013) Global burden of disease attributable to mental and substance use disorders: findings from the Global Burden of Disease Study 2010. Lancet (London, England) 382(9904): 1575-1586.

13. Erskine HE, Whiteford HA, Pike KM (2016) The global burden of eating disorders. Current opinion in psychiatry 29(6): 346-353.

14. Colton PA, Olmsted MP, Daneman D, Farquhar JC, Wong H, et al. (2015) Eating Disorders in Girls and Women with Type 1 Diabetes: A Longitudinal Study of Prevalence, Onset, Remission, and Recurrence. Diabetes care 38(7): 1212-1217.

15. Doyle EA, Quinn SM, Ambrosino JM, Weyman K, Tamborlane WV, et al. (2017) Disordered Eating Behaviors in Emerging Adults with Type 1 Diabetes: A Common Problem for Both Men and Women. Journal of pediatric health care: official publication of National Association of Pediatric Nurse Associates \& Practitioners 31(3): 327-333.

16. Baechle C, Castillo K, Strassburger K, Stahl-Pehe A, Meissner T, et al. (2014) Is disordered eating behavior more prevalent in adolescents with early-onset type 1 diabetes than in their representative peers? Int J Eat Disord 47(4): 342-352.

17. Davidson J (2014) Diabulimia: how eating disorders can affect adolescents with diabetes. Nursing standard (Royal College of Nursing (Great Britain): 1987) 29(2): 44-49.

18. Pinar R (2005) Disordered eating behaviors among Turkish adolescents with and without Type 1 diabetes. Journal of pediatric nursing 20(5): 383-388.

19. Young V, Eiser C, Johnson B, Brierley S, Epton T, et al. (2013) Eating problems in adolescents with Type 1 diabetes: a systematic review with meta-analysis. Diabet Med 30(2): 189-198. 


\section{Current Research in Diabetes \& Obesity Journal}

20. Simon J, Schmidt U, Pilling S (2005) The health service use and cost of eating disorders. Psychol Med 35(11): 1543-1551.

21. Herpertz S, Albus C, Wagener R, Kocnar M, Wagner R, et al. (1998) Comorbidity of diabetes and eating disorders. Does diabetes control reflect disturbed eating behavior? Diabetes care 21(7): 1110-1116.

22. Wilfley D, Berkowitz R, Goebel-Fabbri A, Hirst K, Ievers-Landis C, et al. (2011) Binge eating, mood, and quality of life in youth with type 2 diabetes: baseline data from the today study. Diabetes care 34(4): 858-860.

23. Nielsen S, Emborg C, Molbak AG (2002) Mortality in concurrent type 1 diabetes and anorexia nervosa. Diabetes care 25(2): 309-12.

24. Everson-Rose SA, Meyer PM, Powell LH, Pandey D, Torrens JI, et al. (2004) Depressive symptoms, insulin resistance, and risk of diabetes in women at midlife. Diabetes care 27(12): 2856-2862.

25. Merwin RM, Dmitrieva NO, Honeycutt LK, Moskovich AA, Lane JD, et al. (2015) Momentary Predictors of Insulin Restriction Among Adults With Type 1 Diabetes and Eating Disorder Symptomatology. Diabetes care 38(11): 2025-2032.

26. Clarke DM, Currie KC (2009) Depression, anxiety and their relationship with chronic diseases: a review of the epidemiology, risk and treatment evidence. The Medical journal of Australia 190(S7): S54-60.

27. Engum A (2007) The role of depression and anxiety in onset of diabetes in a large population-based study. J Psychosom Res 62(1): 31-38.

28. Lloyd CE, Dyer PH, Barnett AH (2000) Prevalence of symptoms of depression and anxiety in a diabetes clinic population. Diabetic medicine: a journal of the British Diabetic Association 17(3): 198-202.

29. Braun DL, Sunday SR, Halmi KA (1994) Psychiatric comorbidity in patients with eating disorders. Psychological medicine 24(4): 859-867.

30. Bulik CM, Sullivan PF, Fear JL, Joyce PR (1997) Eating disorders and antecedent anxiety disorders: a controlled study. Acta psychiatrica Scandinavica 96(2):101-117.

31. Pollice C, Kaye WH, Greeno CG, Weltzin TE (1997) Relationship of depression, anxiety, and obsessionality to state of illness in anorexia nervosa. Int J Eat Disord 21(4): 367-376.

32. Strandberg RB, Graue M, Wentzel-Larsen T, Peyrot M, Rokne B (2014) Relationships of diabetes-specific emotional distress, depression, anxiety, and overall well-being with $\mathrm{HbA1c}$ in adult persons with type 1 diabetes. J Psychosom Res 77(3): 174-179.

33. Staite E, Zaremba N, Macdonald P, Allan J, Treasure J, et al. (2018) 'Diabulima' through the lens of social media: a qualitative review and analysis of online blogs by people with Type 1 diabetes mellitus and eating disorders. Diabetic medicine: a journal of the British Diabetic Association 35(10): 1329-1336.

34. Raffi AR, Rondini M, Grandi S, Fava GA (2000) Life events and prodromal symptoms in bulimia nervosa. Psychological medicine 30(3): 727-731.

35. Polivy J, Herman CP (2002) Causes of eating disorders. Annu Rev Psychol 53: 187-213.

36. Heatherton TF, Baumeister RF (1991) Binge eating as escape from selfawareness. Psychol Bull 110(1): 86-108.

37. Takii M, Komaki G, Uchigata Y, Maeda M, Omori Y, et al. (1999) Differences between bulimia nervosa and binge-eating disorder in females with type 1 diabetes: the important role of insulin omission. J Psychosom Res 47(3): 221-231.

38. Mekary RA, Giovannucci E, Willett WC, van Dam RM, Hu FB (2012) Eating patterns and type 2 diabetes risk in men: breakfast omission, eating frequency, and snacking. The American journal of clinical nutrition 95(5): 1182-1189.

39. Gorin AA, Niemeier HM, Hogan P, Coday M, Davis C, et al. (2008) Binge eating and weight loss outcomes in overweight and obese individuals with type 2 diabetes: results from the Look AHEAD trial. Archives of general psychiatry 65(12): 1447-1455.

40. Anderson BJ, Vangsness L, Connell A, Butler D, Goebel-Fabbri A, et al (2002) Family conflict, adherence, and glycaemic control in youth with short duration Type 1 diabetes. Diabetic medicine: a journal of the British Diabetic Association 19(8): 635-642.

41. Powers MA, Richter SA, Ackard DM, Cronemeyer C (2016) Eating disorders in persons with type 1 diabetes: A focus group investigation of early eating disorder risk. J Health Psychol 21(12): 2966-2976.

42. De Paoli T, Rogers PJ (2018) Disordered eating and insulin restriction in type 1 diabetes: A systematic review and testable model. Eating disorders 26(4): 343-360

43. Goebel-Fabbri AE (2009) Disturbed eating behaviors and eating disorders in type 1 diabetes: clinical significance and treatment recommendations. Current diabetes reports 9(2): 133-139.

44. Hastings A, McNamara N, Allan J, Marriott M (2016) The importance of social identities in the management of and recovery from 'Diabulimia': A qualitative exploration. Addictive behaviors report 4: 78-86.

45. Macdonald P, Kan C, Stadler M, De Bernier G L, Hadjimichalis A, et al (2018) Eating disorders in people with Type 1 diabetes: experiential perspectives of both clients and healthcare professionals. Diabet Med 35(2): 223-231.

46. Tierney S, Deaton C, Whitehead J (2009) Caring for people with type 1 diabetes mellitus engaging in disturbed eating or weight control: a qualitative study of practitioners' attitudes and practices. J Clin Nurs 18(3): 384-390.

47. Wilding JP (2014) The importance of weight management in type 2 diabetes mellitus. Int J Clin Pract 68(6): 682-691.

48. Dingemans AE, Bruna MJ, van Furth EF (2002) Binge eating disorder: a review. Int J Obes Relat Metab Disord 26(3): 299-307.

49. Leonard D, McDermott R, Odea K, Rowley KG, Pensio P, et al. (2002) Obesity, diabetes and associated cardiovascular risk factors among Torres Strait Islander people. Australian and New Zealand journal of public health 26(2): 144-159.

50. Valery PC, Ibiebele T, Harris M, Green AC, Cotterill A (2012) Diet, physical activity, and obesity in school-aged indigenous youths in northern australia. Journal of obesity 893508.

51. McCabe MP, Ricciardelli L, Mellor D, Ball K (2005) Media influences on body image and disordered eating among indigenous adolescent Australians. Adolescence 40(157): 115-127.

52. McHugh C, Balaratnasingam S, Campbell A, Chapman M (2017) Suicidal ideation and non-fatal deliberate self-harm presentations in the Kimberley from an enhanced police-mental health service notification database. Australas Psychiatry 25(1): 35-39.

53. Becker AE (2004) Television, disordered eating, and young women in Fiji: negotiating body image and identity during rapid social change. Cult Med Psychiatry 28(4): 533-559.

54. Hay PJ, Carriage C (2012) Eating disorder features in indigenous Aboriginal and Torres Strait Islander Australian peoples. BMC public health 12: 233 . 

CC This work is licensed under Creative
Your next submission with Juniper Publishers will reach you the below assets

- Quality Editorial service

- Swift Peer Review

- Reprints availability

- E-prints Service

- Manuscript Podcast for convenient understanding

- Global attainment for your research

- Manuscript accessibility in different formats

( Pdf, E-pub, Full Text, Audio)

- Unceasing customer service

Track the below URL for one-step submission https://juniperpublishers.com/online-submission.php 